\section{SCIENCE CHINA \\ Technological Sciences}

Special Issue: Celebrating the 100th Anniversary of Harbin Institute of Technology

-Editorial•
August 2020 Vol. 63 No. 8: 1323-1324

https://doi.org/10.1007/s11431-020-1693-2

\title{
Preface: In honor of the 100th Anniversary of Harbin Institute of Technology
}

Harbin Institute of Technology (HIT) was established in 1920 in Harbin, Heilongjiang, China. In 1954, HIT became one of China's first six leading universities. Presently HIT is a member of China's top nine University Union (C9). It is a National Key University with science and engineering as its core and has developed with management, liberal arts, economy, law and other disciplines. This year HIT celebrates its 100th anniversary. HIT's three campuses have graduated more than 300000 students. With strong capabilities in research and innovation, HIT will continue to explore new frontiers and scale greater heights.

HIT was the first in China to establish a school of astronautics, the first university to independently develop a small satellite, and the first to realize the scientific experiment of man-machine coordination on in-orbit maintenance (the Tiangong-2 space manipulator). It built the world's first micro-satellite (Longjiang-2) to complete Earth-to-Moon transfer, near-moon braking, and circumlunar flight, and was the first in China to achieve satellite-ground laser link communications. It was a pioneer in realizing target and multi-beam laser auto-alignment of large laser device, as well as space application of magnetic focusing Hall thruster. It developed China's first computer to play chess and talk to humans, China's first new type long range ocean detection radar system, and its first arc- and spot-welding robot. It was the first to reveal the structure of a ligase complex in the HIV virus. It conducted the world's first spaceflight on-orbit demonstration of a flexible solar array system based on shape memory polymer composites.

HIT has produced a large number of achievements that have contributed to major space missions, including the first launches of the Long March 7 and the Long March 5, as well as the Tiangong-2 space lab and the Shenzhou-11 manned spacecraft.

HIT has signed exchange and cooperation agreements with 278 universities in 39 countries, and since 2011, has led more than 180 international collaborative research projects. In 2011, HIT initiated and jointly established the Association of Sino-Russian Technical Universities (ASRTU) that now counts 67 elite universities as its members. In 2018, HIT became a member of the University of the Arctic, the largest international academic organization in the Circumpolar North.

HIT's expansion has seen the growth of its campuses in line with regional development. In the coastal city of Weihai in East China, HIT opened a campus in 1985, focusing on developing marine research and ocean economy, as well as intelligent manufacturing and smart cities. It has undertaken more than 1000 projects, including ocean detection technologies, unmanned vehicles, intelligent robots, new information technology, cyber security, new materials, water purification, and renewable energy, generating hundreds of patents.

Seizing the opportunity of the growth of the Guangdong-Hong Kong-Macao Greater Bay Area, HIT's Shenzhen campus is becoming a hub for scientific and technological innovation. Starting from the HIT Shenzhen Graduate School in 2002, it has since established comprehensive degree programmes. Its 10 schools and 4 research centres focus on research ranging from materials and artificial intelligence, to space science and bio-pharmaceuticals. It is striving to be an international, high-skilled, and research-oriented campus, by revolutionizing higher education and enhancing international collaboration.

With a "one university, three campuses" model and an expanding international network, HIT is striving to reach its goal of becoming a world-class university. 
HIT in numbers: 22 schools, 86 undergraduate major programmes, 9 national key disciplines, 7 state key laboratories, 38 members of the Chinese Academy of Sciences or the Chinese Academy of Engineering. 11 disciplines are ranked among the global top 1\% according to ESI (Essential Science Indicators) data, with materials science and computer science ranking among the top $1 \%$, engineering discipline ranking among the global top 1\%oo, according to ESI data. In the 2020 ranking of U.S. News \& World Report, HIT ranked 1st on the list of the "Best Global Universities for Electrical and Electronic Engineering" and 6th on the list of the "Best Global Universities for Engineering", respectively.

In honor of HIT's centennial celebration, this special issue has been organized, including 11 papers in Chinese, and 25 papers in English. It explores the recent research progresses of HIT, including Mechanics, Materials Science and Engineering, Automation \& Electrical and Electronic Engineering, Computer Science and Technology, Aerospace Science and Engineering, Mechatronics Engineering, Chemistry, Electronics and Information Engineering, and Energy Engineering. It would be a view to communication and cooperation with world-wide researchers, to promote the development of fundamental studies and engineering applications in related fields.

We wish to express our sincere appreciation to Prof. Tongyi Zhang, Editor-in-Chief of the journal, and Editor Mr. Shujun Sun for their great efforts and guidance in bringing this issue to fruition.

Considering its long and diverse history of achievements over the past century, HIT expects to see even more success ahead. Work at HIT has changed how we see our world, and we will continue to benefit the world with technology innovation in the next century.

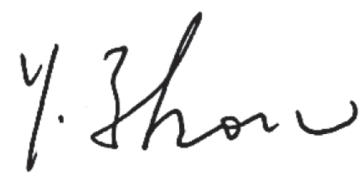

Harbin Institute of Technology July, 2020 